\title{
Prevalencia del uso indebido de alcohol y drogas psicoactivas en un instituto técnico de la ciudad de Babahoyo-Ecuador.
}

\author{
Prevalence of misuse of alcohol and drug psychoactive a technical institute of the city of Babahoyo - \\ Ecuador.
}

\author{
John Flores Moran (*) \\ RESUMEN
}

Se presenta los resultados del estudio descriptivo de prevalencia de alcohol y drogas psicoactivas, en una muestra de 278 estudiantes entre las edades de 14 a 17 años que estudiaban en jornadas matutina y vespertina del Instituto Técnico Eugenio Espejo durante el año lectivo 2011-2012, y se realizó con financiamiento de la Universidad Técnica de Babahoyo, Ecuador. Se aplicó un cuestionario impersonal, similar al utilizado por el Sistema de Datos Uniformes del Observatorio Interamericano de Drogas, con la finalidad de conocer los niveles de prevalencia de vida, del último año y los recientes 30 días. Se encontró que el alcohol fue la sustancia de mayor nivel de consumo. Además, se estudió la presencia de factores de protección ante el consumo de sustancias, relacionados con la preocupación de los padres por la marcha de los estudiantes, y el control que ejercían cuando estaban en casa. Así mismo, se estudió la percepción de riesgo de los estudiantes y sus posibilidades de consumo.

Palabras claves: Alcohol, drogas, factores de protección, percepción de riesgo, prevalencia.

\section{SUMMARY}

The results of descriptive study of prevalence of alcohol and psychoactive drugs is presented, in a sample of 278 students between the ages of 14 to 17 years studying in morning and evening sessions of Eugenio Espejo Technical Institute during the 2011-2012 school year, and it was conducted with funding from the Technical University of Babahoyo, Ecuador. An impersonal, similar to that used by the Data System of the Inter-American Observatory on Drugs, in order to know the levels of lifetime, last year and the recent 30 days questionnaire was applied. It was found that alcohol was the substance of higher level of consumption. Furthermore, the presence of protective factors studied to substance, related to parental concern for the progress of students, and their control when they were at home. Likewise, the risk perception of students and their consumption possibilities were studied.

Keywords: Alcohol, drugs, protection factors, risk perception, prevalence.

\section{INTRODUCCIÓN}

Los estudios descriptivos de prevalencia en el campo del uso indebido de alcohol y drogas psicoactivas, constituye un tema recurrente en la literatura mundial, lo que permite auscultar la presencia cuantitativa del problema en la juventud que se forma en las instituciones de educación media; y a partir de estos datos delinear estrategias de prevención y tratamiento para esta problemática ligada a la salud, educación y con repercusiones en el campo de la seguridad pública.

Desde esta perspectiva, las tasas de prevalencia relacionadas al consumo de estas sustancias, permiten aportan con datos concretos desde las poblaciones 
que se estudian, para que se transformen en insumos objetivos que respalden programas de prevención y erradicación de estos consumos. Programas orientados a salvaguardar el bienestar de un sector tan sensible como el de la juventud, que está en vías de formación y desarrollo.

Estudios locales como el presente, vinculados al Instituto Eugenio Espejo como institución educativa representativa de la ciudad de Babahoyo, dotan de información confiable, de primera mano, sobre la problemática de estudio en su realidad cotidiana. En caso contrario, para poder dimensionarla, solo quedaría revisar las encuestas nacionales que realizan, en conjunto, el Consejo Nacional de Control de Estupefacientes y Sustancias Psicotrópicas (CONSEP); el Observatorio Nacional de Drogas, la Comisión Interamericana para el control del abuso de drogas (CICAD-OEA); la Oficina de las Naciones Unidas contra la droga y el delito (ONUDD); y de aquí extrapolar resultados referenciales por aproximación.

En Ecuador, los últimos estudios de prevalencia, sobre consumo de drogas en estudiantes de enseñanza media, se remontan a la tercera encuesta nacional realizada en el año 2008, en forma coordinada, entre el CONSEP, OND, CICAD-OEA, y ONUDD; donde se menciona que: "la constitución ya concebía el tema de las adicciones como un problema de salud pública, y por ende otorgaba al estado un rol protagónico para su intervención preventiva en conjunto con la comunidad" (Paredes, 2008: 7).

El estudio nacional abordó una población de estudiantes comprendidos entre 15 y 19 años y más; aplicó el cuestionario estandarizado del Sistema de Datos Uniformes (SIDUC) del OND y de la CICADOEA. Los resultados reportados por esta investigación han sido tomados para realizar el presente estudio. A saber, a nivel nacional, la prevalencia de vida, reciente (últimos 12 meses) y actual (últimos 30 días) del consumo de cigarrillos se registra en $46.0 \%, 24.9 \%$ y $14.4 \%$, respectivamente. En cuanto al consumo de alcohol, la prevalencia de vida reciente (últimos 12 meses) y actual (últimos 30 días) se registra en $79.4 \%, 57.5 \%$ y $35.5 \%$, respectivamente. En relación al consumo de drogas, el nivel de prevalencia de vida para marihuana fue de $6.4 \%$, inhalantes $5.7 \%$, cocaína $2.2 \%$ y pasta básica el 1\%, respectivamente. El 17.5\% expresaba curiosidad por probar alguna droga ilícita, pero solo el 5.4\% lo haría si tuvieran la ocasión.
En el año 2012, se realizó la cuarta encuesta nacional, coordinada por el CONSEP y el OND, su metodología, buscó: "Lograr una mayor comprensión del fenómeno drogas, desde una perspectiva más amplia e inclusiva, el OND ha implementado un análisis de categorización de los usos de drogas que deja de lado el análisis clásico de prevalencia como eje de estudio" (OND-CONSEP, 2012. p. 18).

\section{METODOLOGÍA.}

\section{Participantes.}

El estudio se llevó a cabo en el Instituto Técnico Mixto Eugenio Espejo de la ciudad de Babahoyo, que funciona en jornada matutina y vespertina, respectivamente. Se trabajó con estudiantes cuyas edades estaban entre 14 y 17 años. Después de obtener la información estadística de los alumnos matriculados en el periodo lectivo 2011-2012, independientemente del curso de estudios, se conformó la muestra con 278 estudiantes, con el $95 \%$ de confianza y un error de diseño del 5\%, aplicando la fórmula de proporciones, donde el valor de aceptación (p) fue de 0,5. El 68.3\% de la muestra estudiaba en jornada matutina y el $31.7 \%$ en jornada vespertina; de género masculino el $55.4 \%$, y el $48.6 \%$ femenino. El diseño muestral fue en 2 etapas: en la primera se seleccionó a los estudiantes de las especializaciones comprendidas en las edades del estudio (muestreo estratificado) y mediante proporcionalidad se seleccionó el tamaño de la muestra por especialización. En la segunda etapa, se seleccionaron los cursos de las especializaciones; y usando la nómina de los estudiantes, mediante muestreo aleatorio simple, se seleccionó a los estudiantes que contestaron el cuestionario de la investigación.

\section{Instrumento.}

Se aplicó un cuestionario impersonal, que tomó como base el utilizado por el SIDUC y el OND. También se consideró el formato de la encuesta internacional para estudiantes de enseñanza media, utilizado en el año 2005, y se complementó con la información proporcionada por los estudiantes y docentes a través de la aplicación de un grupo focal. El instrumento recogió información referido a niveles de prevalencia estimados en: alguna vez en la vida (prevalencia de vida), últimos doce meses (prevalencia de un año) y últimos treinta días (prevalencia actual) de consumo de cigarrillos, alcohol, drogas ilícitas comunes, factores de protección familiar, percepción de disponibilidad para conseguir drogas, y posibilidad de consumo. 


\section{Procedimiento.}

La aplicación de la encuesta se realizó en el salón de actos del colegio, previa coordinación y autorización de sus autoridades. También se contó con la colaboración de varios docentes asignados para esta tarea. Una vez convocados los estudiantes por grupos, se les habló sobre el estudio: sus objetivos y sobre la confidencialidad de la información recogida por los instrumentos aplicados. Se entregaron los cuestionarios, se explicó sus características y luego los estudiantes procedieron a su llenado respectivo y posterior entrega.

\section{RESULTADOS.}

Acontinuación se presentan los resultados obtenidos del cuestionario aplicado a los 278 estudiantes.

Tabla No 1 . Descripción de la muestra.

\begin{tabular}{lll}
\hline EDADES & f & $\mathbf{\%}$ \\
\hline 14 años & 117 & 42,09 \\
15 años & 106 & 38,13 \\
16 años & 36 & 12,95 \\
17 años & 19 & 6,83 \\
Total & $\mathbf{2 7 8}$ & $\mathbf{1 0 0 , 0 0}$ \\
\hline
\end{tabular}

En la tabla $\mathrm{N}^{\circ} 1$ se presenta la información sobre la edad de la muestra. Se puede apreciar que el mayor porcentaje se ubica en 14 años, y el porcentaje menor en 17 años.

En relación a los niveles de prevalencia de alcohol y de drogas ilícitas, se presentan los datos organizados por prevalencia de vida (Tabla 2) del año (Tabla 3) y los recientes 30 días (Tabla 4).

De las drogas investigadas a nivel de prevalencia de vida, el consumo de alcohol se presenta como

Tabla $\mathbf{N}^{\circ} 2$. Prevalencia de vida.

\begin{tabular}{|c|c|c|c|}
\hline TIPODE DROGA & f & $\%$ & $\begin{array}{l}\text { EDAD DE } \\
\text { INICIO }\end{array}$ \\
\hline Cigarrillo & 63 & 22,66 & 12-13 Años \\
\hline Alcohol & 132 & 47,48 & 13-14 Años \\
\hline Marihuana & 8 & 2,88 & \\
\hline Inhalantes & 5 & 1,80 & \\
\hline Cocaína & 6 & 2,16 & \\
\hline Pasta básica & 7 & 2,52 & \\
\hline No reporta & 57 & 20,52 & \\
\hline Total & 278 & 100,00 & \\
\hline
\end{tabular}

altamente significativo en este grupo estudiantil: el $47.48 \%$ de la muestra expresa haberlo consumido alguna vez en su vida, y la edad de inicio de consumo está comprendida entre 13 y 14 años; mientras que las drogas psicoactivas no presentan datos significativos.

Tabla $N^{\circ}$ 3. Prevalencia del año de consumo.

\begin{tabular}{lcc}
\hline TIPO DE DROGA & f & $\mathbf{\%}$ \\
\hline Cigarrillo & 24 & 8,63 \\
Alcohol & 74 & 26,62 \\
Marihuana & 4 & 1,44 \\
Inhalantes & 2 & 0,72 \\
Pasta básica & 3 & 1,08 \\
No reporta & 168 & 60,43 \\
Total & $\mathbf{2 7 8}$ & $\mathbf{1 0 0 , 0 0}$ \\
\hline
\end{tabular}

A nivel de prevalencia del año, el consumo de alcohol arroja el mayor porcentaje (26,62\%), sigue siendo lo más significativo, pues de los 132 alumnos que reportaron su consumo en la prevalencia de vida, 74 de ellos mantuvieron su consumo durante el último año.

Tabla No 4. Prevalencia de 30 días.

\begin{tabular}{lrc}
\hline TIPO DE DROGA & f & \% \\
\hline Cigarrillo & 23 & 8,27 \\
Alcohol & 35 & 12,59 \\
Marihuana & 4 & 1,44 \\
Inhalantes & 2 & 0,72 \\
Cocaína & 3 & 1,08 \\
Pasta básica & 3 & 1,08 \\
No reporta & 208 & 74,82 \\
Total & $\mathbf{2 7 8}$ & $\mathbf{1 0 0 , 0 0}$ \\
\hline
\end{tabular}

En la tabla 4 se observa que del grupo de 74 estudiantes que declararon haber consumido alcohol en el último año, el $12.59 \%$ mantuvieron su consumo en los 30 días anteriores a la aplicación del cuestionario; y probablemente, tengan o hayan empezado a establecer una relación de adicción con esta sustancia.

Tabla $\mathbf{N}^{\circ}$ 5. Días que se han emborrachado los estudiantes con prevalencia en los últimos 30 días.

\begin{tabular}{lcc}
\hline $\begin{array}{l}\text { DIAS QUE SE HAN } \\
\text { EMBORRACHADO }\end{array}$ & f & $\mathbf{\%}$ \\
\hline 1 & 22 & 62,85 \\
2 & 5 & 14,29 \\
3 & 3 & 8,57 \\
No reporta & 5 & 14,29 \\
Total & $\mathbf{3 5}$ & $\mathbf{1 0 0 , 0 0}$ \\
\hline
\end{tabular}


De 35 estudiantes que declaran prevalencia reciente, 22 de ellos se ha emborrachado una vez al mes, cinco lo han hecho por dos ocasiones, y tres de ellos por un espacio de tres días.

Tabla $\mathbf{N}^{\circ}$ 6. Preocupación de los padres respecto a la marcha de sus hijos en el colegio.

\begin{tabular}{lrc}
\hline PREOCUPACIÓN & \multicolumn{1}{c}{ f } & \% \\
\hline Mucho & 149 & 53,6 \\
Bastante & 57 & 20,5 \\
Poco & 55 & 19,8 \\
Nada & 17 & 6,1 \\
Total & $\mathbf{2 7 8}$ & $\mathbf{1 0 0 , 0}$
\end{tabular}

El $19.8 \%$ de los estudiantes encuestados percibe que la preocupación de sus padres, en relación a la situación del colegio y por ende de la marcha en sus estudios, es poca; mientras que el $6.1 \%$ considera que no hay nada o ninguna de preocupación.

Tabla $\mathbf{N}^{\circ}$ 7. Control de los padres, cuando pasan en casa.

\begin{tabular}{lrc}
\hline CATEGORIAS & f & \% \\
\hline Siempre saben dónde están. & 179 & 64,4 \\
A veces saben dónde están. & 84 & 30,2 \\
Nunca saben dónde están. & 15 & 5,4 \\
Total & $\mathbf{2 7 8}$ & $\mathbf{1 0 0 , 0}$ \\
\hline
\end{tabular}

Al ser encuestados en relación a si sus padres saben dónde están cuando pasan en casa, el 5.4\% (15 estudiantes) manifiestan que en general nunca lo saben. Dato que guarda relación con el 6.1\% (17 estudiantes) cuyos padres no se preocupan de cómo marchan sus hijos en el colegio, tal como se muestra en la tabla 6.

Tabla $N^{\circ}$ 8. Percepción de riesgo en relación al consumo de drogas.

\begin{tabular}{lll}
\hline PREGUNTAS & f & \% NO \\
\hline $\begin{array}{l}\text { ¿Crees que las personas exageran } \\
\text { cuando dicen que bajo ningún }\end{array}$ & & \\
pretexto se debería probar drogas & 240 & 86,33 \\
ilícitas? & & \\
$\begin{array}{l}\text { ¿Crees que consumir drogas ilícitas } \\
\text { de vez en cuando no es peligroso? }\end{array}$ & 160 & 57,55 \\
\hline
\end{tabular}

En la tabla 8 se tiene que el $86.33 \%$ no considera exagerado el mensaje preventivo en relación a las drogas, probablemente de adultos (como padres o docentes). El $57.55 \%$ cree que no es peligroso
Tabla $\mathbf{N}^{\circ}$ 9. Posibilidad de consumo de drogas.

\begin{tabular}{lccc}
\hline \multicolumn{1}{c}{ PREGUNTAS } & f & \% SI \\
\hline $\begin{array}{l}\text { ¿Crees que se debería tener una } \\
\text { experiencia con las drogas ilícitas? }\end{array}$ & 28 & 10,07 \\
$\begin{array}{l}\text { ¿Probarías drogas ilícitas para saber } \\
\text { qué se siente? }\end{array}$ & 11 & 3,96 \\
$\begin{array}{l}\text { ¿Aceptarías si un amigo te ofreciera } \\
\text { drogas? }\end{array}$ & 3 & 1,07 \\
\hline
\end{tabular}

consumir drogas de vez en cuando. Al contrastar estos resultados con sus creencias personales (Tabla 9), por ejemplo, el $57.55 \%$ considera que consumir drogas de vez en cuando no es peligroso; pero en la práctica, solo el 3,96\% (11 estudiantes) las probaría para saber que se siente.

\section{DISCUSIÓN.}

Al explorar el consumo de las diferentes sustancias, se encontró que el alcohol, tiene el mayor nivel de prevalencia de vida, con un $47.5 \%$, y su consumo en los últimos 30 días involucra a 35 estudiantes, de los cuales 22 de ellos se han emborrachado una vez al mes.

Estos resultados coinciden con la tercera encuesta nacional del CONSEP sobre uso indebido de drogas en el 2008. En sus conclusiones, afirma que el alcohol es la sustancia más consumida por los estudiantes ecuatorianos; además, reporta que el primer consumo se realiza a los 12.8 años; datos similares se encontró en el presente estudio: el inicio del consumo se ubica en el rango de 13 a 14 años. Si estos datos forman parte de una realidad actual, llama la atención que las campañas de prevención gubernamentales se sigan orientando hacia el consumo de sustancias psicoactivas, y no hacia el consumo del alcohol; tampoco hacen la diferencia como para atacar su consumo de forma específica.

La cuarta encuesta nacional realizada por el CONSEP y el OND, en relación al consumo del alcohol, refiere que el $20.56 \%$ de los estudiantes están inmersos en lo que se denomina uso ocasional, es decir, salir a beber en las últimas dos semanas, cinco vasos en una sola sentada, pero sin especificar el tipo de bebida alcohólica.

Existen resultados similares encontrados en esta investigación con los encontrados en las encuestas nacionales de que los cigarrillos son la segunda sustancia de mayor consumo entre los estudiantes. 
En cuanto a la presencia de factores de protección vinculados a la familia, y específicamente a la presencia de los padres, este estudio determinó que el $6.1 \%$ de los estudiantes manifiesta que sus padres no conocen nada de la marcha educativa de sus hijos, y un $5.4 \%$ manifiesta que sus padres nunca saben dónde están, cuando se encuentran en casa. Resulta contradictorio, como menciona Cabrera (2012), que siendo la familia el primer espacio de socialización y proveedor de protección, estas relaciones familiares sean cada vez más frágiles y con vínculos poco claros. Aun cuando, como señala el Observatorio Chileno de Drogas (2012), la presencia de los padres, constituye un factor de protección clave para inhibir conductas de riesgo.

La percepción de riesgo en relación al consumo de drogas, evidencia que aún existe un $57.55 \%$ de estos estudiantes, con los que se debe trabajar en jornadas de prevención para contrarrestar futuras experiencias con drogas.

En cuanto a la posibilidad de consumo, se encontró que un $10.07 \%$ de los estudiantes cree que debería tener una experiencia con drogas, solo un $1.07 \%$ la aceptaría si se la ofrecen. Resultados que contrastan con los reportados del CONSEP el 2008, donde se reporta que el $17.5 \%$ tendría una experiencia con drogas y el 5.4\% sí consumiría en la primera oportunidad que tuviera.

En suma, los resultados obtenidos en este estudio, confirman la prevalencia del alcohol como la sustancia de mayor consumo entre los adolescentes de 14 a 17 años. Esto sugiere que la toma de acciones preventivas deben ser focalizadas por parte de organizaciones gubernamentales o privadas encargadas de esta problemática. Problemática cuyas consecuencias tal vez estén enmascaradas, no se notan de forma inmediata en los jóvenes; es posible que no revistan el sensacionalismo mediático de la heroína; pero, su cronicidad puede dejar secuelas que trastornen muchos proyectos de vida juveniles.
En tal sentido, el fortalecimiento de los factores de protección debería constituir uno de los objetivos importantes a tener en cuenta por los entes encargados. Los datos de la percepción de riesgo y la posibilidad de consumo, siguen confirmando la necesidad de mantener campañas de prevención desde las instituciones educativas; no solo en el nivel medio, sino también a nivel superior, tal como está asignado a los departamentos de bienestar universitario desde la Ley Orgánica de Educación Superior (LOES).

\section{REFERENCIAS BIBLIOGRÁFICAS.}

1. Cabrera, K. (2012). Niños y adolescentes "gomeros" de la calle. Un caso de Quito. Universitas. Revista de Ciencias Sociales y Humanas. $\quad \mathrm{N}^{\circ} 17$. Universidad Politécnica del Ecuador.

2. Paredes (2008). Citado en: Tercera encuesta nacional sobre el consumo de drogas en estudiantes de enseñanza media. Consejo Nacional de Control de Sustancias Estupefacientes y Psicotrópicas. Observatorio Nacional de drogas. Ecuador.

3. CONSEJO NACIONAL DE CONTROL DE SUSTANCIAS ESTUPEFACIENTES Y PSICOTRÓPIAS. OBSERVATORIO NACIONAL DE DROGAS (2008). Tercera encuesta nacional sobre el consumo de drogas en estudiantes de enseñanza media. Ecuador.

4. OBSERVATORIO CHILENO DE DROGAS (2012). Consumo de marihuana en población escolar: Análisis de prevalencia e incidencia.

5. CONSEJO NACIONAL DE CONTROL DE SUSTANCIAS ESTUPEFACIENTES Y PSICOTRÓPIAS. OBSERVATORIO NACIONAL DE DROGAS (2013). Cuarta encuesta nacional sobre uso de drogas en estudiantes de 12 a 17 años. Ecuador.

6. PROGRAMA MUNDIAL DE EVALUACIÓN DEL USO INDEBIDO DE DROGAS (2003). Encuestas escolares sobre el uso indebido de drogas.

7. Encuesta internacional para estudiantes de enseñanza media (2005). Cuestionario estandarizado. 